\title{
GENERALIZED LOCAL FATOU THEOREMS AND AREA INTEGRALS
}

\author{
B. A. MAIR, STAN PHILIPP AND DAVID SINGMAN
}

\begin{abstract}
Let $X$ be a space of homogeneous type and $W$ a subset of $X \times$ $(0, \infty)$. Then, under minimal conditions on $W$, we obtain a relationship between two modes of convergence at the boundary $X$ for functions defined on $W$. This result gives new local Fatou theorems of the Carleson-type for solutions of Laplace, parabolic and Laplace-Beltrami equations as immediate consequences of the classical results. Lusin area integral characterizations for the existence of limits within these more general approach regions are also obtained.
\end{abstract}

\section{INTRODUCTION}

Recently, the classical Fatou theorems for solutions of the Laplace equation on $\mathbf{R}^{n} \times \mathbf{R}_{+}$(cf. [12]), solutions of certain general parabolic equations on $\mathbf{R}^{n} \times \mathbf{R}_{+}$and solutions of the heat equation on the right half-space (cf. [11]) have been improved to allow sequential approaches to the boundary at any prescribed degree of tangency. Also, the well-known Koranyi approach region for PoissonSzegö integrals on the generalized upper half-space has been improved (cf. [14]).

In the above-mentioned papers, the approach regions were restricted by a certain cross-sectional area condition at every height. However, it is clear that the existence of limits at the boundary within a region $\Omega$ only depends on the structure of $\Omega$ very close to the boundary.

Also, the Fatou theorems were all obtained by the method in [12], which consists of two main stages. First, a certain "sup" maximal function was shown to be weak-type $(1,1)$ and then the Fatou theorem was obtained from properties of the kernel appearing in the various integral representations. The approach adopted in this paper deviates from this method, especially in the second stage.

In this paper we consider an upper half-space of the form $X \times \mathbf{R}_{+}$where $X$ is a group which is also a space of homogeneous type. We introduce the notion of a locally admissible subset of $X \times \mathbf{R}_{+}$which satisfies a cross-sectional area condition close to the boundary.

Received by the editors November 1, 1987 and, in revised form, November 10, 1988. Presented at the meeting on Potential Theory, Faculty of Mathematics \& Physics, Charles University, Praha, Czechoslovakia, July 19-24, 1987.

1980 Mathematics Subject Classification (1985 Revision). Primary 31B25, 42B25; Secondary $32 \mathrm{~A} 40$. 
Now, from the necessary and sufficient results in $[12,14,11]$, it is clear that we cannot obtain weak-type inequalities for the "sup" maximal function over these more general locally admissible regions. This difficulty is avoided by considering the corresponding "lim sup" maximal functions over the locally admissible regions. The proof of the weak-type inequality in [14] is adapted to obtain the corresponding result for our "lim sup" maximal function.

Then in $\S 3$ we obtain a differentiation theorem by a slight variation of the standard methods (cf. [13, 11]). This result also incorporates the analog of widening the aperture of a cone, for these more general approach regions.

The "standard regions" defined in this paper are just abstractions of the usual cones, parabolic, two-sided parabolic and Koranyi regions which appear as the approach regions in the classical Fatou theorems. So, from these well-known Fatou theorems, we know that various functions have standard limits (limits within the standard regions).

The second stage in the method presented here consists of using the differentiation theorem obtained in $\S 3$ to deduce the existence of limits within the more general locally admissible regions from the existence of standard limits.

More specifically, in $\S 4$ we prove that if $W \subset X \times \mathbf{R}_{+}$and $E \subset X$ are such that for each $y \in E, W$ contains a standard region with vertex at $y$, then standard and locally admissible limits are equivalent almost everywhere on $E$, for any function defined on $W$. This result therefore yields new local Fatou theorems (cf. [2, 3, 5, 6, 7, 8, 9, 10]) without any extra effort.

In the last section we consider area integrals where the integration takes place on locally admissible regions. There we show that finiteness of these area integrals is equivalent to existence of standard limits a.e., which is equivalent to existence of limits within locally admissible regions almost everywhere.

\section{A WEAK-TYPE INEQUALITY}

For the reader's convenience, we define what we mean by a space of homogeneous type (cf. [4, 14]).

Definition 2.1. $(X, \rho, \mu)$ is a space of homogeneous type if $X$ is a topological space, $\rho: X \times X \rightarrow[0, \infty)$ and $\mu$ is a positive Borel measure on $X$ satisfying

(i) $\rho(x, y)=\rho(y, x)$ for all $x, y \in X$.

(ii) $\rho(x, y)=0 \Leftrightarrow x=y$.

(iii) (Triangle Inequality) There exists a constant $\gamma>0$ such that

$$
\rho(x, z) \leq \gamma[\rho(x, y)+\rho(y, z)], \quad \text { for all } x, y, z \in X .
$$

The $\rho$-ball of center $x$ and radius $r>0$, denoted by $B(x, r)=\{y \in X: \rho(y, x)$ $<r\}$, satisfies

(iv) $\{B(x, r): r>0\}$ is a base of open neighborhoods of each $x \in X$.

(v) (Doubling Property) There exists a constant $A>0$ such that

$$
\mu(B(x, 2 r)) \leq A \mu(B(x, r)) \text { for all } x \in X, r>0 \text {. }
$$


This doubling property implies that for each $\alpha>0$ there exists $C(\alpha)>0$ such that

$$
\mu(B(x, \alpha r)) \leq C(\alpha) \mu(B(x, r)) \text { for all } x \in X, r>0 .
$$

For any $\Omega \subset X \times \mathbf{R}_{+}$, the cross-section at height $t$ is

$$
\Omega(t)=\{x \in X:(x, t) \in \Omega\} .
$$

Definition 2.2. Let $(X, \rho, \mu)$ be a space of homogeneous type, $\Omega \subset X \times \mathbf{R}_{+}$ and $y \in X$.

(i) $\Omega$ is said to be locally $\alpha$-admissible at $y$ if $(y, 0)$ is a limit point of $\Omega$ and there exists $\Omega^{\prime} \supset \Omega$ such that

(a) $\mu\left(\Omega^{\prime}(t)\right)=0(\mu(B(y, t)))$ as $t \rightarrow 0_{+}$,

(b) $(z, s) \in \Omega, \rho(x, z)<\alpha(t-s) \Rightarrow(x, t) \in \Omega^{\prime}$.

(ii) $\Omega$ is locally admissible at $y$ if $\Omega$ is locally $\alpha$-admissible for some $\alpha>0$.

If $\omega \subset \mathbf{R}^{n} \times \mathbf{R}_{+}$is such that for some $T>0, \omega \cap\left(\mathbf{R}^{n} \times(0, T)\right)$ is the truncation of some admissible region (as defined in [11]), then $\omega$ is locally admissible.

Now, assume $X$ is a group with identity $e$ and $(X, \rho, \mu)$ is a space of homogeneous type. For any $\Omega \subset X \times \mathbf{R}_{+}$having $(e, 0)$ as a limit point and $f \in L^{1}(X, \mu)$, define

$$
M_{\Omega}^{*} f(y)=\limsup _{\Omega \ni(x, t) \rightarrow(e, 0)} \frac{1}{\mu(B(y x, t))} \int_{B(y x, t)}|f| d \mu .
$$

As in [13], we show that $M_{\Omega}^{*}$ is weak-type $(1,1)$ under certain invariance conditions on $\mu$. In order to do this, we need the following covering lemma (cf. $[4,14])$.

Lemma 2.1. Let $(X, \rho, \mu)$ be a space of homogeneous type and $E \subset X$. If $\{B(x, r(x)): x \in E\}$ is a cover of $E$ with $\sup _{x \in E} r(x)<+\infty$, then there exists a countable subcollection $\left\{B\left(x_{k}, r_{k}\right): k=1,2, \ldots\right\}, r_{k}=r\left(x_{k}\right)$ such that

(i) $\left\{B\left(x_{k}, r_{k}\right)\right\}$ are pairwise disjoint, and

(ii) for each $x \in E$ there exists $k \in \mathbf{N}$ such that $r(x)<2 r_{k}$ and $x \in$ $B\left(x_{k}, 4 \gamma r_{k}\right)$.

This follows from the proof of Théorème 1.2, Chapitre III in [4]. For a space of homogeneous type $(X, \rho, \mu)$, if $X$ is a group, $\rho$ is left-invariant if $\rho(z x, z y)=\rho(x, y)$ for all $x, y, z \in X$ and $\mu$ is left-invariant if $\mu(x E)=$ $\mu(E)$ for every $x \in X$ and Borel $E \subset X$.

Theorem 2.1. Let $X$ be a group with identity $e$ and $(X, \rho, \mu)$ a space of homogeneous type such that $\rho, \mu$ are left-invariant and $\mu\left(E^{-1}\right)=\mu(E)$ for every Borel $E \subset X$. If $\Omega$ is locally $\alpha$-admissible at $e$ then there exists a constant $c>0$ such that

$$
\mu\left(\left\{x \in X: M_{\Omega}^{*} f(x)>\lambda\right\}\right) \leq \frac{c}{\lambda}\|f\|_{1}
$$

for all $\lambda>0, f \in L^{1}(X, \mu)$. 
Proof. This is similar to the proof of Theorem 1.5 in [14]. Since $\Omega$ is locally admissible, there exist constants $M, T>0$ such that

$$
\mu\left(\Omega^{\prime}(t)\right) \leq M \mu(B(e, t)) \text { for all } 0<t<T .
$$

Let $\beta=\min (\alpha, 2 \gamma)$ and fix $R>0$ such that $8 \beta^{-1} \gamma R<T$. For each $\lambda>0$, let

$$
E_{\lambda}=\left\{x \in X: \sup _{0<r<R} \frac{1}{\mu(B(x, r))} \int_{B(x, r)}|f| d \mu>\lambda\right\} .
$$

For each $x \in E_{\lambda}$, let

$$
r(x)=\sup \left\{r: 0<r<R, \frac{1}{\mu(B(x, r))} \int_{B(x, r)}|f| d \mu>\lambda\right\}
$$

Then,

$$
\mu(B(x, r(x))) \leq \frac{1}{\lambda} \int_{B(x, r(x))}|f| d \mu .
$$

$\{B(x, r(x)): x \in E\}$ is a cover of $E$ and $r(x) \leq R$ for all $x \in E$; hence by the covering lemma we have a sequence $B\left(x_{k}, r_{k}\right), r_{k}=r\left(x_{k}\right)$ satisfying (i) and (ii) in Lemma 1.3.

Now, let $M_{\Omega}^{*} f\left(x_{0}\right)>\lambda$. Then there exists $(y, s) \in \Omega$ such that

$$
\frac{1}{\mu\left(B\left(x_{0} y, s\right)\right)} \int_{B\left(x_{0} y, s\right)}|f| d \mu>\lambda
$$

and $s<R$.

Hence $x_{0} y \in E_{\lambda}$. So there exists $k \in \mathbf{N}$ such that $x_{0} y \in B\left(x_{k}, 4 \gamma r_{k}\right)$ and $r\left(x_{0} y\right)<2 r_{k}$.

Now, by the definition of $r\left(x_{0} y\right), s<r\left(x_{0} y\right)$. Hence $s<r\left(x_{0} y\right)<2 r_{k}<$ $4 \beta^{-1} \gamma r_{k}$, by choice of $\beta$. Since $\beta<\alpha, \Omega$ is locally $\beta$-admissible. Hence,

$$
\begin{aligned}
\rho\left(x_{0}^{-1} x_{k}, y\right) & =\rho\left(x_{0} y, x_{k}\right)<4 \gamma r_{k} \\
& =\beta\left[\left(4 \beta^{-1} \gamma r_{k}+s\right)-s\right]<\beta\left[8 \beta^{-1} \gamma r_{k}-s\right] .
\end{aligned}
$$

Since $(y, s) \in \Omega$, it follows that

$$
x_{0}^{-1} x_{k} \in \Omega^{\prime}\left(8 \beta^{-1} \gamma r_{k}\right)
$$

and so

$$
x_{0} \in x_{k}\left[\Omega^{\prime}\left(8 \beta^{-1} \gamma r_{k}\right)\right]^{-1} .
$$


Therefore

$$
\begin{aligned}
\mu\left(\left\{M_{\Omega}^{*} f>\lambda\right\}\right) & \leq \sum_{k=1}^{\infty} \mu\left(x_{k}\left[\Omega^{\prime}\left(8 \beta^{-1} \gamma r_{k}\right)\right]^{-1}\right) \\
& =\sum_{k=1}^{\infty} \mu\left(\Omega^{\prime}\left(8 \beta^{-1} \gamma r_{k}\right)\right) \\
& \leq M \sum_{k=1}^{\infty} \mu\left(B\left(e, 8 \beta^{-1} \gamma r_{k}\right)\right), \quad \text { since } 8 \beta^{-1} \gamma R<T \\
& \leq M C\left(\beta^{-1} \gamma\right) \sum_{k=1}^{\infty} \mu\left(B\left(e, r_{k}\right)\right) \\
& =M C\left(\beta^{-1} \gamma\right) \sum_{k=1}^{\infty} \mu\left(B\left(x_{k}, r_{k}\right)\right) \\
& \leq \frac{M C\left(\beta^{-1} \gamma\right)}{\lambda} \sum_{k=1}^{\infty} \int_{B\left(x_{k}, r_{k}\right)}|f| d \mu \\
& =\frac{M C\left(\beta^{-1} \gamma\right)}{\lambda} \int_{\bigcup_{k=1}^{\infty} B\left(x_{k}, r_{k}\right)}|f| d \mu \\
& \leq \frac{M C\left(\beta^{-1} \gamma\right)}{\lambda}\|f\|_{1} .
\end{aligned}
$$

\section{A DIFFERENTIATION THEOREM}

Throughout the remainder of this paper, $X$ is a group with identity $e$ and $(X, \rho, \mu)$ is a space of homogeneous type such that $\rho, \mu$ are left-invariant and $\mu\left(E^{-1}\right)=\mu(E)$ for every Borel $E \subset X$.

The following differentiation theorem can be proved by a slight variation of the standard method (cf. $[13,11]$ ).

Theorem 3.1. If $\Omega \subset X \times \mathbf{R}_{+}$is locally admissible at $e$ and $\nu$ is a signed regular Borel measure on $X$, then

$$
\lim _{\Omega \ni(x, t) \rightarrow(e, 0)} \frac{\nu(B(y x, t))}{\mu(B(y x, t))}=\frac{d \nu}{d \mu}(y),
$$

for $\mu$-a.e. $y \in X$.

This result can be improved to take widenings of aperture into account as follows.

Definition 3.1. For any $\Omega \subset X \times \mathbf{R}_{+}$and $a>0$, define

$$
\Omega_{a}=\{(x, t):(x, a t) \in \Omega\} .
$$

Then, if $S(y ; \alpha)=\{(x, t): \rho(x, y)<\alpha t\}$,

$$
(S(y ; \alpha))_{a}=S(y ; a \alpha) .
$$


Hence $\Omega_{a}$ is called the widening of $\Omega$ by a multiple of $a$.

The following result is an immediate consequence of the doubling condition and the definitions.

Proposition 3.1. If $\Omega$ is locally $\alpha$-admissible at $y$ then $\Omega_{a}$ is locally a $\alpha$ admissible at $y$.

Proposition 3.2. Let $\Omega$ be locally $\alpha$-admissible at $e$ and $\Omega^{\prime}$ be as in Definition 1.2. Then there exists $\Sigma \subset X \times \mathbf{R}_{+}$such that

(i) $\Omega \subset \Sigma \subset \Omega^{\prime}$.

(ii) $\Sigma$ is locally admissible at $e$.

(iii) $(x, t) \in \Sigma, t<s \Rightarrow(x, s) \in \Sigma$.

(iv) $0<a<b \Rightarrow \Sigma(a) \subset \Sigma(b)$ and $\Sigma_{a} \subset \Sigma_{b}$.

Proof. Fix $\beta>\min \left(\alpha, \gamma^{-1} \alpha\right)$ and define

$$
\Sigma=\{(x, t): \rho(x, y) \leq \beta(t-s), \text { for some }(y, s) \in \Omega\} .
$$

(i) $\Omega \subset \Sigma$ is clear.

$$
\begin{aligned}
(x, t) \in \Sigma & \Rightarrow \rho(x, y) \leq \beta(t-s) \text { for some }(y, s) \in \Omega \\
& \Rightarrow \rho(x, y)<\alpha(t-s) \text { and }(y, s) \in \Omega \\
& \Rightarrow(x, t) \in \Omega^{\prime} .
\end{aligned}
$$

(ii) Let $(y, s) \in \Sigma$ and $\rho(x, y)<\beta(t-s)$. Then there exists $\left(y_{0}, s_{0}\right) \in \Omega$ such that

$$
\rho\left(y, y_{0}\right) \leq \beta\left(s-s_{0}\right) .
$$

Hence

$$
\begin{aligned}
\rho\left(x, y_{0}\right) & \leq \gamma\left[\rho(x, y)+\rho\left(y, y_{0}\right)\right] \\
& <\gamma\left[\beta(t-s)+\beta\left(s-s_{0}\right)\right] \\
& <\alpha\left(t-s_{0}\right) .
\end{aligned}
$$

Therefore $(x, t) \in \Omega^{\prime}$. Hence $\Sigma$ is locally $\beta$-admissible at $e$. (iii) and (iv) are clear.

Hence we can always assume that our locally admissible regions satisfy conditions (ii), (iii), (iv) in Proposition 3.2.

Definition 3.2. Let $\Omega \subset X \times \mathbf{R}_{+}$have $(e, 0)$ as a limit point. A function $u$ is said to have $\Omega$-limit $\lambda$ at $y \in X$ if $u(x, t) \rightarrow \lambda$ as $(x, t)$ approaches $(y, 0)$ inside $y \Omega_{a}$ for every $a>0$.

Theorem 3.2. Let $\Omega$ be locally admissible at $e$ and $\nu$ be a signed regular Borel measure on $X$. Then

$$
\frac{\nu(B(x, t))}{\mu(B(x, t))} \text { has } \Omega \text {-limit } \frac{d \nu}{d \mu}(y) \text { for } \mu \text {-a.e. } y \in X \text {. }
$$


Proof. By Propositions 3.1 and 3.2 we can apply Theorem 3.1 to each widening $\Sigma_{N}, N=1,2,3, \ldots$, where $\Sigma$ is as in Proposition 3.2. This gives a set $E_{N}$ such that $\mu\left(E_{N}\right)=0$ and for all $y \notin E_{N}$,

$$
\lim _{y \Sigma_{N} \ni(x, t) \rightarrow(y, 0)} \frac{\nu(B(x, t))}{\mu(B(x, t))}=\frac{d \nu}{d \mu}(y) .
$$

By (iv) of Proposition 2.4, we obtain, for every $a>0$,

$$
\lim _{y \Sigma_{N} \ni(x, t) \rightarrow(y, 0)} \frac{\nu(B(x, t))}{\mu(B(x, t))}=\frac{d \nu}{d \mu}(y),
$$

if $y \notin \bigcup_{N=1}^{\infty} E_{N}$.

\section{STANDARD AND $\Omega$-Limits}

In this section we obtain our main result which shows that limits within a locally admissible region are equivalent $\mu$-almost everywhere to limits within the standard regions $S(y ; \alpha)$, for any function. This enables us to deduce the improved Fatou theorems in $[12,14,11]$ from their classical counterparts and to obtain new local Fatou theorems without any extra effort.

The main lemma is a generalization of a technique of Calderón (cf. [2]) and the main theorem is motivated by a result of Brelot and Doob (cf. [1]) concerning fine and nontangential limits.

We continue to assume that $X$ is a group with identity $e,(X, \rho, \mu)$ is a space of homogeneous type where $\rho, \mu$ are left-invariant and $\mu\left(E^{-1}\right)=\mu(E)$ for every Borel $E \subset X$.

Definition 4.1. (1) For each $y \in X$ and $\alpha>0$, let

$$
S(y ; \alpha)=\left\{(x, t) \in X \times \mathbf{R}_{+}: \rho(x, y)<\alpha t\right\},
$$

called the standard region with vertex $y$ and aperture $\alpha$.

(2) A function $u$ is said to have standard limit $\lambda$ at $y \in X$ if $u(x, t) \rightarrow \lambda$ as $(x, t)$ approaches $(y, 0)$ inside $S(y, \alpha)$ for every $\alpha>0$.

(3) For each $r>0$ and $y \in X$, let $U(y ; r)=B(y ; r) \times(0, r)$.

(4) For any $\Omega \subset X \times \mathbf{R}_{+}$which has $(y, 0)$ as a limit point, $\Omega \cap U(y ; r)$ is called a truncation of $\Omega$.

Lemma 4.1. Let $E \subset X$ and $W \subset X \times \mathbf{R}_{+}$be such that $W$ contains some truncation of some standard region with vertex $y$, for each $y \in E$. Let $\Omega$ be locally admissible at $e$. Then for $\mu$-almost every $y \in E, W$ contains some truncation of any widening of $y \Omega$.

Proof. Let $\left\{\alpha_{k}\right\}$ and $\left\{r_{k}\right\}$ be sequences of real number such that $\left\{\alpha_{k}\right\}$ increases to $\infty$ and $\left\{r_{k}\right\}$ decreases to zero. Put

$$
E_{k, j}=\left\{y \in E: S\left(y, \alpha_{k}\right) \cap U\left(y, r_{j}\right) \subset W\right\} .
$$

If we can prove the lemma for $E$ replaced by $E_{k, j}$, since $E=\bigcup_{k, j} E_{k, j}$, we will have it for $E$. Thus we assume that there exists $\alpha, r>0$, independent of 
$y$, such that

$$
S(y ; \alpha) \cap U(y ; r) \subset W,
$$

for all $y \in E$. Choose integer $N \geq 1$ such that $\frac{1}{N}<r$. For each $m \geq N$ and $a>0$, let

$$
E_{m}(a)=\left\{y \in E:\left(y \Omega_{a}\right) \cap U\left(y ; \frac{1}{m}\right) \subset W\right\} .
$$

Let $D$ denote the set of points $y \in E$ for which

$$
\lim _{y \Omega_{a} \ni(x, t) \rightarrow(y, 0)} \frac{\mu(E \cap B(x, t))}{\mu(B(e, t))}=1, \quad \text { for all } a>0 .
$$

Then, from Theorem 3.2, $\mu(E \backslash D)=0$. It clearly suffices to prove that $D \subset$ $\bigcup_{m=N}^{\infty} E_{m}(a)$, for each $a>0$. To do this, let $y \notin \bigcup_{m=N}^{\infty} E_{m}(a)$ for some $a>0$. Then there is a sequence of points $\left\{\left(x_{m}, t_{m}\right): m \geq N\right\}$ such that

$$
\left(x_{m}, t_{m}\right) \in\left(y \Omega_{a}\right) \cap U\left(y ; \frac{1}{m}\right), \quad\left(x_{m}, t_{m}\right) \notin W .
$$

Hence,

(i) $\left(y^{-1} x_{m}, a t_{m}\right) \in \Omega$,

(ii) $\rho\left(x_{m}, y\right)<\frac{1}{m}, t_{m}<\frac{1}{m}$,

(iii) $\left(x_{m}, t_{m}\right) \notin W$.

From (i), $\left(y^{-1} x_{m}, \alpha t_{m}\right) \in \Omega_{\alpha^{-1} a}$; hence from (ii)

$$
\left(x_{m}, \alpha t_{m}\right) \rightarrow(y, 0) \quad \text { within } y \Omega_{\alpha^{-1} a} .
$$

So, to see that $y \notin D$, it suffices to show that

$$
\mu\left(E \cap B\left(x_{m}, \alpha t_{m}\right)\right)=0 \text { for } m \geq N .
$$

To do this, let $z \in E$. Then $S(z ; \alpha) \cap U\left(y ; \frac{1}{m}\right) \subset W$ for all $m \geq N$. From (iii) and (ii) above, $\left(x_{m}, t_{m}\right) \notin S(z ; \alpha)$. Hence $\rho\left(x_{m}, z\right) \geq \alpha t_{m}$. So $E \cap$ $B\left(x_{m}, \alpha t_{m}\right)=\varnothing$.

Theorem 4.1. Let $E \subset X$ and $W$ be a subset of $X \times \mathbf{R}_{+}$which contains some truncation of a standard region with vertex $y$ for each $y \in E$. Let $\Omega$ be locally admissible at $e$ and $u$ a function on $W$ which has standard limit $\psi(y)$ at every $y \in E$. Then $u$ has $\Omega$-limit $\psi(y)$ at $\mu$-almost every $y \in E$.

Proof. For brevity, put

$$
S(y ; \alpha, \delta)=S(y ; \alpha) \cap(B(y, \alpha \delta) \times(0, \delta))
$$

for $y \in X, \alpha>0, \delta>0$. As in Lemma 4.1, we can assume that the standard regions contained in $W$ with vertices on $E$ have uniform aperture and truncations. That is, there exist $\alpha, \delta>0$, independent of $y$, such that

$$
S(y ; \alpha, \delta) \subset W, \quad \text { for each } y \in E .
$$

We shall actually obtain the same conclusion under the seemingly weaker assumption that $u$ has limit $\psi(y)$ with $S(y ; \alpha)$ for every $y \in E$.

Then for each $y \in E$ and $m \in \mathbf{N}$, there exists a positive rational $\delta_{m}(y)$ such that

$$
|u(x, t)-\psi(y)|<\frac{1}{m} \quad \text { for all }(x, t) \in S\left(y ; \alpha, \delta_{m}(y)\right) \text {. }
$$


By dividing $E$ into a countable number of subsets, we can assume that $\delta_{m}(y)=$ $\delta_{m}$ for all $y \in E$.

Now, let

$$
Q_{m}(y)=\bigcup\left\{S\left(z ; \alpha, \delta_{m}\right): z \in E, S\left(z ; \alpha, \delta_{m}\right) \cap S\left(y ; \alpha, \delta_{m}\right) \neq \varnothing\right\} .
$$

Claim 1.

$$
Q_{m}(y) \supset \bigcup\left\{S\left(z ; \alpha, \delta_{m}\right): z \in E \cap B\left(y, \alpha \delta_{m}\right)\right\} .
$$

To see this, let $z \in B\left(y, \alpha \delta_{m}\right)$. Then

$$
\rho(z, y)<\alpha r_{m}<\alpha \delta_{m}
$$

Putting $t=r_{m}$ we obtain

$$
\rho(z, y)<\alpha t, \quad \rho(z, y)<\alpha \delta_{m}, \quad t<\delta_{m} .
$$

That is,

$$
(z, t) \in S\left(y ; \alpha, \delta_{m}\right)
$$

Hence

$$
S\left(y ; \alpha, \delta_{m}\right) \cap S\left(z ; \alpha, \delta_{m}\right) \neq \varnothing .
$$

Now, let $\nu_{m}<\min \left(\delta_{m},(2 \gamma)^{-1} \delta_{m}\right)$. Then

Claim 2.

$$
Q_{m}(y) \supset W_{m} \cap\left(B\left(y, \alpha \nu_{m}\right) \times\left(0, \nu_{m}\right)\right)
$$

where

$$
W_{m}=\bigcup_{z \in E} S\left(z ; \alpha, \delta_{m}\right)
$$

Let

$$
(x, t) \in W_{m} \cap\left(B\left(y, \alpha \nu_{m}\right) \times\left(0, \nu_{m}\right)\right) .
$$

Then there exists $z \in E$ such that $(x, t) \in S\left(z ; \alpha, \delta_{m}\right)$. Hence

$$
\begin{aligned}
\rho(z, y) & \leq \gamma[\rho(z, x)+\rho(x, y)]<\gamma\left[\alpha t+\alpha \nu_{m}\right] \\
& =\gamma \alpha\left(t+\nu_{m}\right)<2 \gamma \alpha \nu_{m}<\alpha \delta_{m} .
\end{aligned}
$$

Therefore $z \in B\left(y, \alpha \delta_{m}\right) \cap E$. Hence $(x, t) \in Q_{m}(y)$ by Claim 1. Now, by Lemma 4.1, for each $m$ there exists $D_{m} \subset E$ such that $\mu\left(D_{m}\right)=0$ and for each $z \in E \backslash D_{m}$ and $a>0$ there exists $\tau_{m}=\tau_{m}(z, \alpha)>0$ such that $\tau_{m}<\nu_{m}$ and

$$
z \Omega_{a} \cap\left(B\left(z, \alpha \tau_{m}\right) \times\left(0, \tau_{m}\right)\right) \subset W_{m} .
$$

Put $D=\bigcup_{m=1}^{\infty} D_{m}$. Then $\mu(D)=0$.

Now, let $y \in E \backslash D$ and

$$
(x, t) \in y \Omega_{a} \cap\left(B\left(z, \alpha \tau_{m}\right) \times\left(0, \tau_{m}\right)\right) .
$$

Then

$$
(x, t) \in W_{m} \cap\left(B\left(y, \alpha \nu_{m}\right) \times\left(0, \nu_{m}\right)\right) .
$$


Hence $(x, t) \in Q_{m}(y)$. So there exists $z \in E$ such that

$$
(\xi, s) \in S\left(z ; \alpha, \delta_{m}\right) \cap S\left(y ; \alpha, \delta_{m}\right),
$$

and

$$
(x, t) \in S\left(z ; \alpha, \delta_{m}\right) \text {. }
$$

Hence

$$
|u(x, t)-\psi(z)|<1 / m, \quad|u(\xi, s)-\psi(z)|<1 / m
$$

and

$$
|u(\xi, s)-\psi(y)|<1 / m \text {. }
$$

Therefore

$$
|u(x, t)-\psi(y)|<3 / m \text {. }
$$

\section{ApPLicAtions}

In our first application, we consider $X=\mathbf{R}^{n}$ with the additive group structure, $\rho$ the Euclidean metric, and $\mu$ Lebesgue measure on $\mathbf{R}^{n}$. Then by using the classical result in [3] and Theorem 4.1, we obtain

Theorem 5.1. Let $E \subset \mathbf{R}^{n}$ and $W$ be a subset of $\mathbf{R}^{n} \times \mathbf{R}_{+}$which contains $a$ truncated cone $W_{y}$ with vertex $y$ for each $y \in E$. Let $\Omega$ be locally admissible at the origin. If $u$ is a solution of the Laplace equation on $W$ which is upper or lower bounded on $W_{y}$ for each $y \in E$, then $u$ has finite $\Omega$-limits at Lebesgue almost every point of $E$.

This generalizes the results in $[12,11]$.

For the second application, consider $X=\mathbf{R}^{n}$ with the additive group structure, $\rho(x, y)=\|x-y\|^{2}$, where $\|\cdot\|$ is Euclidean norm, and $\mu$ be Lebesgue measure. Let $L$ be a second-order linear parabolic operator in divergence form on $\mathbf{R}^{n} \times(0, T)$. Then, under certain general conditions on the coefficients of $L$ (cf. [9]) we obtain the following result by combining the local Fatou theorem in [9] and Theorem 4.1.

Theorem 5.2. Let $E \subset \mathbf{R}^{n}$ and $W$ be a subset of $\mathbf{R}^{n} \times(0, T)$ which contains a truncated parabolic region $W_{y}$ with vertex $y$ for each $y \in E$. Let $\Omega$ be locally admissible at the origin. If $L u=0$ on $W$ and $u$ is either upper or lower bounded on $W_{y}$ for each $y \in E$, then $u$ has finite $\Omega$-limits at Lebesgue almost every point of $E$.

A similar result can be obtained for solutions of the heat equation on the right half-space, thus generalizing results in $[10,11]$.

In our final application we consider $X$ to the Heisenberg group $\mathbf{C}^{n} \times \mathbf{R}$ with the group operation

$$
(z, t) \cdot(w, s)=(z+w, t+s+2 \operatorname{Im}\langle z, w\rangle),
$$


where

$$
\langle z, w\rangle=\sum_{k=1}^{n} z_{k} \bar{w}_{k} .
$$

Let $\rho((z, t),(w, s))=\left(\|z-w\|^{4}+|t-s|^{2}\right)^{1 / 4}$ and $\mu$ be Lebesgue measure (cf. $[5,7,14])$.

The results in $[8,7,5]$ concerning the boundary behavior for "harmonic" functions on $X \times \mathbf{R}_{+}$(identified with the generalized upper half-plane) give immediate improvements by applying Theorem 4.1 (cf. [14]).

\section{Area integrals}

The classical Lusin area integral characterizations of standard limits (cf. [5, $6,13])$ can be extended to the case where the integration is performed over locally admissible regions.

To be specific we only consider the case of Laplace harmonic functions on $\mathbf{R}_{+}^{n+1}$.

Definition 6.1. For any truncated locally admissible region $\Omega \subset \mathbf{R}_{+}^{n+1}$ having $(0,0)$ as a limit point and harmonic $u$ on $\mathbf{R}_{+}^{n+1}$, define the area integral

$$
S_{\Omega} u(y)=\left(\int_{y+\Omega} \int|\nabla u(x, t)|^{2} t^{1-n} d x d t\right)^{1 / 2}
$$

for $y \in \mathbf{R}^{n}$.

It is well known (cf. [13]) that if $\Omega$ is a truncated cone,

$$
\Gamma_{\alpha}(0, \delta)=\{(x, t):|x|<\alpha t, t<\delta\},
$$

we have

Theorem 6.1. Let $u$ be harmonic on $\mathbf{R}_{+}^{n+1}$.

(i) $u$ has a finite nontangential limit at each

$$
y \in E \subset \mathbf{R}^{n} \Rightarrow \text { for a.e. } y \in E, S_{\Gamma_{\alpha}(0, \delta)} u(y)<\infty
$$

for every $\alpha>0, \delta>0$.

(ii) Conversely, if for each $y \in E \subset \mathbf{R}^{n}$, there exists $\alpha>0, \delta>0$ such that

$$
S_{\Gamma_{\alpha}(0 ; \delta)} u(y)<\infty
$$

then $u$ has finite nontangential limits a.e. on $E$.

To extend this result, we define the following.

Definition 6.2. Let $u$ be defined on $\mathbf{R}_{+}^{n+1}$.

(i) Let $\Omega$ be locally admissible at $(0,0)$ containing a truncation of some standard region with vertex at $(0,0)$. We say that $u$ has finite $\Omega$-uniform area integral on $E \subset \mathbf{R}^{n}$ if for any $a>0$ and $r>0$,

$$
S_{\Omega_{a}^{r}} u(y)<\infty, \quad \text { for all } y \in E,
$$


where $\Omega_{a}^{r}=\Omega_{a} \cap U(0, r)$ (the truncation of a widening of $\Omega$ ).

(ii) Let $\Omega$ be locally admissible at $(0,0)$ containing a truncation of some standard region with vertex at $(0,0)$. We say $u$ has finite $\Omega$-area integral on $E \subset \mathbf{R}^{n}$ if for each $y \in E$ there exists $a>0$ and $r>0$ such that

$$
S_{\Omega_{a}^{r}} u(y)<\infty
$$

(iii) $u$ has finite locally admissible area integral on a set $E \subset \mathbf{R}^{n}$ if for each $y \in E$ there exists a truncated locally admissible region $\Omega$ containing a truncation of some standard region with vertex at $(0,0)$, having $(0,0)$ as a limit point, such that

$$
S_{\Omega} u(y)<\infty
$$

Remark 6.1. (1) If locally admissible regions were completely determined by aperture (as is the case for cones) then (ii) and (iii) above coincide.

(2) Clearly, if $\Omega$ is locally admissible at $(0,0)$, containing a truncation of some standard region with vertex at $(0,0)$, then $u$ has finite $\Omega$-uniform area integral on $E$ for some $\Omega \Rightarrow u$ has finite $\Omega$-area integral on $E$ for some $\Omega \Rightarrow u$ has finite locally admissible area integral on $E \Rightarrow u$ has finite nontangential limits a.e. on $E$ (by Theorem 6.1).

Now, suppose a harmonic $u$ has a finite nontangential limit at each $y \in E \subset$ $\mathbf{R}^{n}$, where $E$ is compact. Then, in proving the classical theorem $6.1(\mathrm{i})$, it is actually shown that

$$
\int_{R} \int|\nabla u(x, t)|^{2} t d x d t<\infty, \quad \text { where } R=\bigcup_{y \in E} \Gamma_{\alpha}(y ; \delta)
$$

(cf. [13]).

By applying Lemma 4.1 and the cross-sectional area condition on a locally admissible $\Omega$, to the proof in [13], we can show that,

$$
\int_{E} S_{\Omega_{a}^{r}}^{2} u(y) d y \leq C \int_{R} \int|\nabla u(x, t)|^{2} t d x d t .
$$

Hence, by applying Theorem 4.1 we obtain

Theorem 6.2. Let $u$ be harmonic in $\mathbf{R}_{+}^{n+1}$ and $E \subset \mathbf{R}^{n}$. Then the following are equivalent.

(i) $u$ has finite $\Omega$-uniform area integral a.e. on $E$ for some $\Omega$.

(ii) $u$ has finite $\Omega$-area integral a.e. on $E$ for some $\Omega$.

(iii) $u$ has finite locally admissible area integral a.e. on $E$.

(iv) $u$ has finite nontangential limits a.e. on $E$.

(v) $u$ has finite $\Omega$-limits a.e. on $E$ for any $\Omega$ which is locally admissible at $(0,0)$.

Analogous results hold for the heat equation on the upper half-space and the right half-space (cf. [6]), and for the Laplace-Beltrami equation on the generalized upper half-space (cf. [5]). 


\section{REFERENCES}

1. M. Brelot and J. L. Doob, Limites angularies et limites fines, Ann. Inst. Fourier (Grenoble) 13 (1963), 395-415.

2. A. P. Calderon, On the behavior of harmonic functions at the boundary, Trans. Amer. Math. Soc. 68 (1950), 47-54.

3. L. Carleson, On the existence of boundary values for harmonic functions in several variables, Ark. Mat. 4 (1961), 393-399.

4. R. Coifman and G. Weiss, Analyse harmonique non-commutative sur ceriaines espaces homogenes, Lecture Notes in Math., vol. 242, Springer-Verlag, New York and Berlin, 1971.

5. D. Geller, Some results in $H^{p}$ theory for the Heisenberg group, Duke Math J. 47 (1980), 365-390.

6. J R. Hattemer, Boundary behavior of temperatures. I, Studia Math. 25 (1964), 111-155.

7. A. Korányi, Harmonic functions on hermitian hyperbolic space, Trans. Amer. Math. Soc. 135 (1969), 507-516.

8. A. Korányi and R. B. Putz, Local Fatou theorem and area theorem for symmetric spaces of rank one, Trans. Amer. Math. Soc. 224 (1976), 157-168.

9. B. A. Mair, Fine and parabolic limits for solutions of second-order linear parabolic equations on an infinite slab, Trans. Amer. Math. Soc. 284 (1984), 583-599.

10. B_ Boundary behavior of positive solutions of the heat equation on a semi-infinite slab, Trans. Amer. Math. Soc. 295 (1986), 687-697.

11. B. A. Mair and D. Singman, A generalized Fatou theorem, Trans. Amer. Math. Soc. 300 (1987), 705-719.

12. A. Nagel and E. M. Stein, On certain maximal functions and approach regions, Adv. in Math. 54 (1984), 83-106.

13. E. M. Stein, Singular integrals and differentiability properties of functions, Princeton Univ. Press, Princeton, N. J., 1970.

14. J. Sueiro, On maximal functions and Poisson-Szegö integrals, Trans. Amer. Math. Soc. 298 (1986), 653-669.

15. C. C. Tu, Non-tangential limits of a solution of a boundary-value problem for the heat equation, Math. Systems Theory 3 (1969), 130-138.

Department of Mathematics, Texas Tech University, Lubbock, Texas 79409

Department of Mathematics, The Pennsylvania State University, Altoona Campus, Altoona, Pennsylvania 16603 (Current address of Stan Philipp)

Department of Mathematical Science, George Mason University, Fairfax, Virginia 22030 (Current address of David Singman)

Current address (B. A. Mair): Department of Mathematics, University of Florida, Gainesville, Florida 32611 